\title{
Fiber-Based Measurement of Bow-Shock Spectra for Reentry Flight Testing
}

\author{
Timothy Schott ${ }^{1}$, G. C. Herring ${ }^{2}$, and Michelle M. Munk ${ }^{3}$ \\ NASA Langley Research Center,Hampton, Virginia,23681 \\ J. H. Grinstead ${ }^{4}$ \\ NASA Ames Research Center, Moffett Field, California, 94035 \\ and \\ D. K. Prabhu ${ }^{5}$ \\ Eloret, Inc., Moffett Field, California 94035
}

\begin{abstract}
We demonstrated a fiber-based approach for obtaining optical spectra of a glowing bow shock in a high-enthalpy air flow. The work was performed in a ground test with the NASA Ames Aerodynamic Heating Facility (AHF) that is used for atmospheric reentry simulation. The method uses a commercial fiber optic that is embedded in the nose of an ablating bluntbody model and provides a line-of-sight view in the streamwise direction - directly upstream into the hot post-shock gas flow. Both phenolic impregnated carbon ablator (PICA) and phenolic carbon (PhenCarb 28) materials were used as thermal protection systems. Results show that the fibers survive the intense heat and operate sufficiently well during the first several seconds of a typical AHF run $(20 \mathrm{MJ} / \mathrm{kg})$. This approach allowed the acquisition of optical spectra, enabling a Boltzmann-based electronic excitation temperature measurement from $\mathrm{Cu}$ atom impurities (averaged over a line-of-sight through the gas cap, with a 0.04 sec integration time).
\end{abstract}

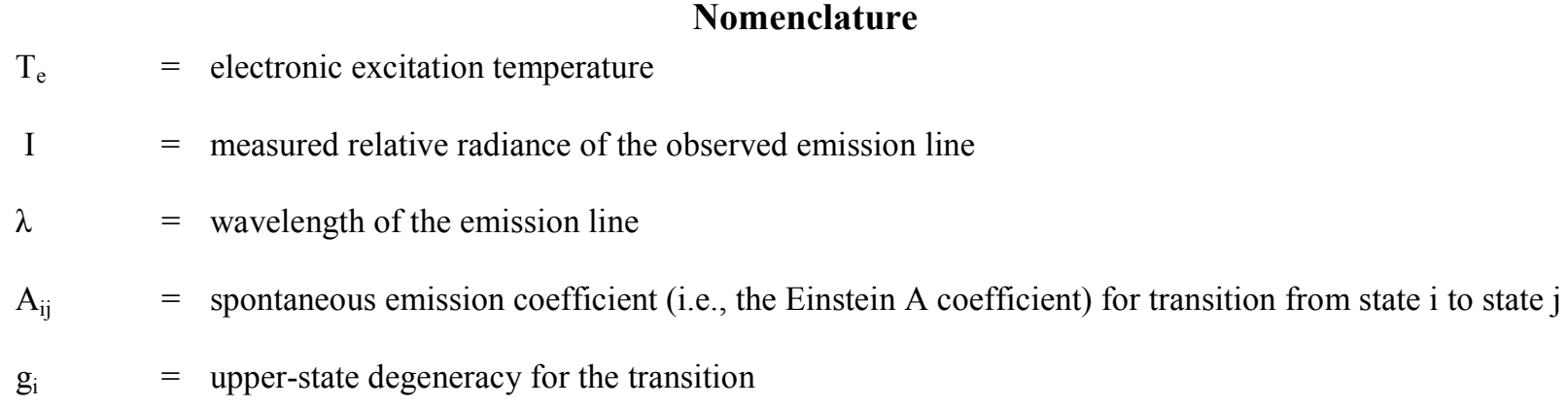

\section{Background}

$\mathrm{R}$ adiometry and spectroradiometry instrumentation for measuring bow shock emission ahead of blunt-body atmospheric entry vehicles was successfully demonstrated in well-known NASA flight experiments in the 1960s as part of the Apollo program. In the Fire I and Fire II flight tests, ${ }^{1,2}$ each entry vehicle had a stacked series of three "clean" beryllium forebody heat shields, two of which were jettisoned successively during reentry. Each heat shield had its own fused quartz window at the stagnation point that provided viewing of the bow shock. The total

\footnotetext{
${ }^{1}$ AST, Flight Systems Remote Sensing Branch, B1202/MS 468

${ }^{2}$ AST, Advanced Sensing and Optical Measurements Branch, B1200/MS493

${ }^{3}$ Technology Development Manager, Atmospheric Flight \& Entry Systems Br., MS489, Senior Member AIAA

${ }^{4}$ Senior Research Scientist, Aerothermodynamics Branch, MS 230-2, Associate Fellow AIAA

${ }^{5}$ Senior Research Scientist, Eloret Corporation, Sunnyvale, CA 94087, MS 230-3, Associate Fellow AIAA
} 
radiometer (a thermopile) shared the optical path with the spectral radiometer in a chassis within the reentry vehicle. The Apollo 4 flight test, ${ }^{3,4}$ also included two thermopile radiometers plug-mounted within the ablating heat shield. The instrument used a very clever stepped conical recess in the heat shield plug, behind which the small (1/10 in.) window was located within a 1 in.-long tube with a $1 / 4$-in. diameter. The window mount incorporated heat sinking to help slow heat transfer to the window that was located $1 \frac{3}{4}$-in. from the surface. The thermopile was located directly behind the window.

The overall objectives of these tests were to characterize the total heating environment for Apollo lunar return missions. The data from Fire II and Apollo 4 have been - and continue to be - analyzed, underscoring the importance of radiometry instrumentation to entry environments research and the development of thermal protection systems (TPS) for entry vehicles. Future entry systems that will encounter a significant amount of radiative heating are most likely to use ablative TPS materials. In such cases the aerothermal environments analysis necessary to size the forebody heat shield will require use of simulation tools that couple shock layer radiation and ablation. ${ }^{5}$ These tools are validated using ground and flight test data.

Advances in photonics technology in the decades since the Apollo era have enabled the development of new approaches to instrumented TPS. One promising approach is the use of optical fibers to transmit the collected radiation to a remote location within the entry vehicle. Miniaturized grating spectrographs coupled to the optical fibers can be used to acquire spectrally resolved shock radiance at data rates on the order of $100 \mathrm{~Hz}$. The wavelength span and spectral resolution of contemporary miniature spectrographs greatly exceed those used during the Apollo era flight tests. The supporting equipment available for fiber optic multiplexing and detection offer many options for implementing a versatile spectroradiometer instrument in an entry vehicle.

\section{Previous Work}

In ground testing, fairly elaborate optical diagnostics ${ }^{6}$ can be used as an important source of off-body flow information from the post-shock non-equilibrium hot gas. ${ }^{7}$ Most ground testing typically uses a transverse probing geometry to view the gas cap emission spectra. ${ }^{8-10}$ Some testing uses streamwise viewing with mirrors instead of fibers. ${ }^{11,12}$ Another study used windowless and purged apertures to extract the optical emission, ${ }^{13}$ which works well for the deep ultraviolet (UV) spectrum.

In flight testing, ${ }^{1-4,} 14,15$ broadband optical radiometry from bow shocks is relatively more common, while spectrally-resolved radiometry is rare and limited in scope. A key problem is penetrating the heat shield for optical access without compromising the integrity of the thermal protection of the heat shield. Hence experimental work is heavily supplemented by ground testing in high-enthalpy facilities that simulate atmospheric reentry. However, flight testing is preferred for gaining maximum confidence in the results.

\section{Present Work}

Since additional flight testing continues to be of interest, we are considering new flight-test strategies. Typical ground-test optical arrangements are not practical for flight tests because of (a) probe geometries that contain a lineof-sight probe volume that is transverse to the freestream flow direction, (b) relatively large apertures in the sensitive thermal protection system materials, or (c) relatively large external spectrometers. In the present work, we extend the ablating-model work of Reference 9 and investigate a rarely-used fiber geometry, ${ }^{16}$ one that provides onaxis streamwise viewing of the gas cap of an ablating model and is suitable for flight during atmospheric reentry.

The demonstration tests had three objectives: (a) prove the feasibility of a new fiber optic-based concept for optical access through a forebody heat shield for radiometer/spectrometer bow shock radiation measurements, (b) examine the transmission characteristics of such an optical fiber in a high enthalpy flow simulating atmospheric entry, and (c) determine a favorable configuration for maximizing the lifetime of the fiber embedded in an ablating TPS material. The paper will discuss experimental details of test models, their optical design, the optical instrumentation and data acquisition system, preliminary results, and modifications for a viable flight instrument.

\section{Arc Jet Facility}

The testing was performed at Ames Research Center (ARC) in the Aerodynamic Heating Facility (AHF), which was fitted with a 7-inch diameter nozzle. ${ }^{17}$ This facility uses Argon-gas sheathed $\mathrm{Cu}$ electrodes in a segmented (or 
constricted) heater with a current of $\sim 2000$ A to heat the airflow to a stagnation temperature of $\sim 7000 \mathrm{~K}$ (centerline enthalpy of $\sim 20 \mathrm{MJ} / \mathrm{kg})^{18}$

\section{Test Models}

A total of 14 test models were used: 11 of which were constructed with a phenolic impregnated carbon ablator (PICA) TPS, while three had a carbon phenolic (PhenCarb 28) TPS. ARC provided the mechanical design of the models as well as fabrication of supporting structural components for the TPS. Langley Research Center (LaRC) designed the instrumentation configurations, machined the ablator material, installed the model instrumentation, and assembled the models. ARC performed the final assembly to a sting adapting skirt used to divert the flow away from the model sting (which normally holds larger models).

As shown in Figure 1, the ablator was attached to an insulating tile made of silicone impregnated refractory ceramic ablator (SIRCA), which was attached to an aluminum backing plate. Alignment dowels were used for stability and to maintain orientation. The ablator measured $2 \mathrm{in}$. in diameter and $1.5 \mathrm{in}$. in thickness, while the insulator was 1 in. thick. The PICA ablator was coated with a thin layer of RTV over its entire perimeter to prevent a path for hot gases to flow laterally through the porous material. This was a lesson learned from Mars Science Laboratory (MSL) Entry, Descent and Landing Instrumentation (MEDLI) arc jet testing with PICA pressure port models. The ablators were fitted with the various fiber optic configurations on a fixture and allowed to cure before model assembly began.

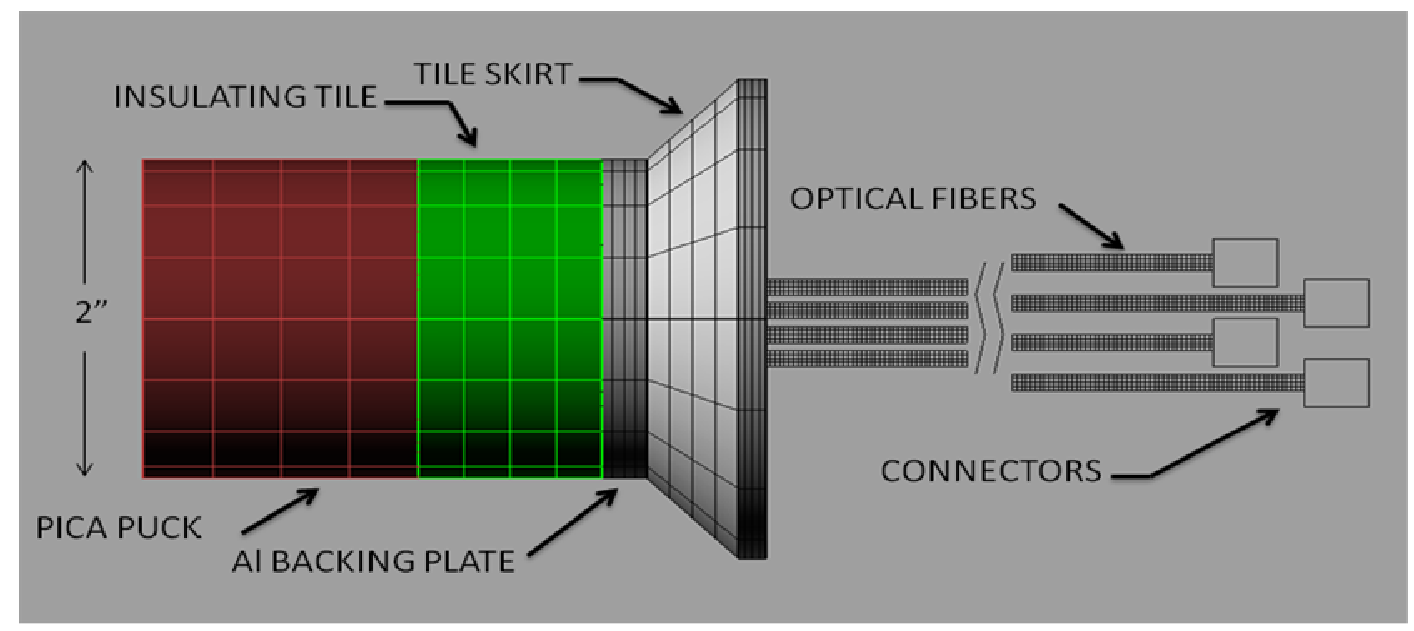

Figure 1. Test model schematic.

\section{Model Assembly}

Fused silica optical fibers with core diameters of 300,400 , and $600 \mu \mathrm{m}$ were installed in the models at depths of $0.10,0.15,0.20,0.25$ and $0.30 \mathrm{in}$. depending on the model. The fibers were approximately $50 \mathrm{in}$. in length and had either FC or SMA connectors. The fibers were installed flush at the bottom of counterbores machined into the face of the models at $15^{\circ}$ from normal, away from the model centerline axis. This was done in accordance with previous work to direct the field of view away from the intense light of the arc heater upstream of the nozzle throat ${ }^{9}$. The counterbores were machined to diameters of $0.10,0.07$, and 0.05 in.. The various counterbore configurations were chosen to find a trend indicating an advantage for maximizing the effective observation lifetime of the fiber. A secondary motive was to correlate results with MSL arc jet tests with the same diameter bores for pressure transducer ports. The maximum fiber depth was dictated by the fiber view angle at the bottom of the cavity. A depth of 0.3 in. results in a small loss of optical energy through vignetting with a $0.10 \mathrm{in}$. diameter opening. 


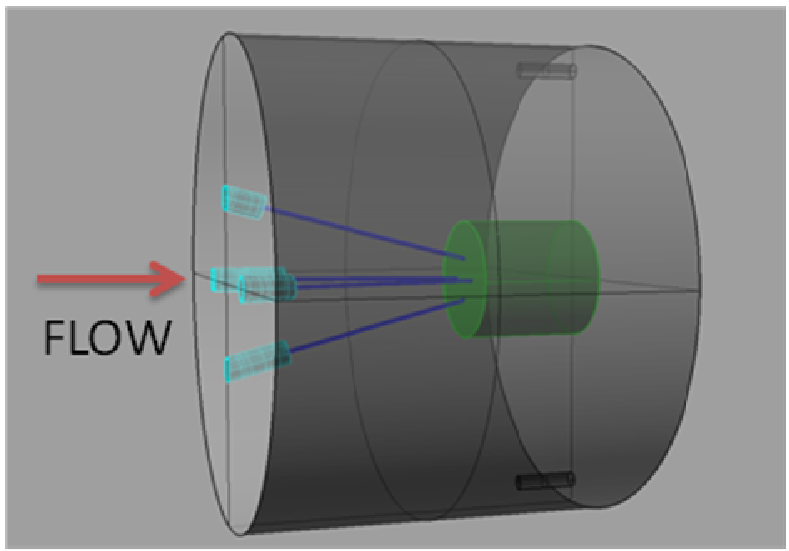

Figure 2a. Ghosted view of a typical PICA puck.

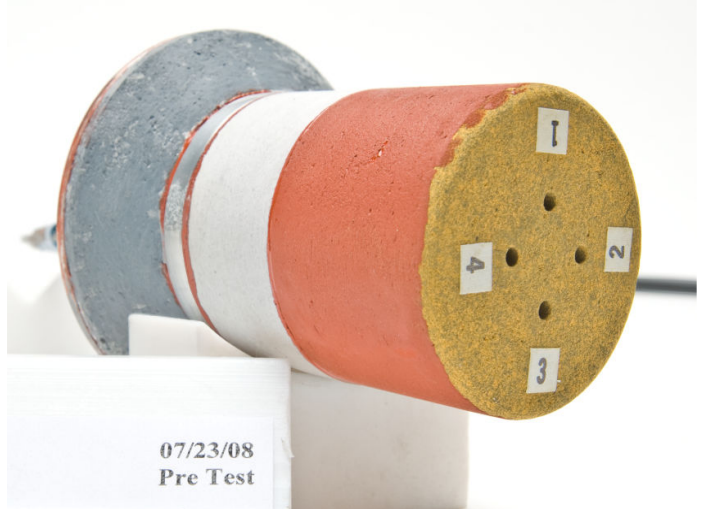

Figure 2b. Photograph of a PICA model.

As depicted in Figure 2a, six of the models were fitted with four fibers located on a circle at $90^{\circ}$ intervals, with the remainder being a mix that included one model with thermocouples (TCs). The fibers were installed from the rear recess of the puck before additional parts were assembled. The fibers were coated with RTV 560 a short distance from the fiber tip before insertion while applying additional adhesive at the insertion point. This allowed the adhesive to be drawn into the model to seal the fiber. After all fibers were installed, the recess was potted with remaining adhesive. The fibers were then inspected visually to ensure that the apertures were flush with the bottom of the counterbores in the model face. The models were placed vertically on laboratory fixtures cut with slotted holes so that the fibers could pass through the slots and left undisturbed for curing. The SIRCA tiles and aluminum backing plates were bored through to allow the fiber connectors to pass since it was not practical to install the connectors after assembly. The parts were then cleaned, primed and bonded together with RTV 560 to complete the assembly. The models were $2 \mathrm{in}$. in diameter and $3.5 \mathrm{in}$. in length (including skirt). Figure $2 \mathrm{~b}$ shows one of the completed models.

Three of the models were particularly challenging to build. Two required special 1-inch diameter plugs. One required blind holes where a very thin wall of residual material at the model face was left in front of the two fibers. Under test, recession would remove the thin wall exposing the fibers at different times (due to different thicknesses of residual material). As shown in Figure 3a, one plug was fitted with a single $300 \mu \mathrm{m}$ fiber with a sapphire window recessed flush with the plug surface above it. The window was $12.7 \mathrm{~mm}$ diameter and $1 \mathrm{~mm}$ thick. The fiber tip can be seen at the bottom of the $0.10 \mathrm{in}$. counterbore with a total depth of $0.29 \mathrm{in} .$. The fiber tip was $\sim 0.05 \mathrm{in}$. from the bottom surface of the window. This is one version of a "storm window" intended to prolong the life of the fiber. Figure $3 \mathrm{~b}$ shows another plug that was fitted with a single $300 \mu \mathrm{m}$ fiber and three type K TCs. The thermocouple plug was fitted with 0.015 in. diameter butt welded TCs at depths of $0.1,0.2$, and 0.45 in., respectively, with leads exiting out the side of the plug. The TCs were installed in a crossing fashion with beads at the center, with the fiber positioned in one of two open quadrants. Because PICA is somewhat electrically conductive, the leads were insulated with alumina tubes which were recessed into grooves down the sides of the plug. Beyond the bottom of the plug, silicone sleeves were used for insulation. 


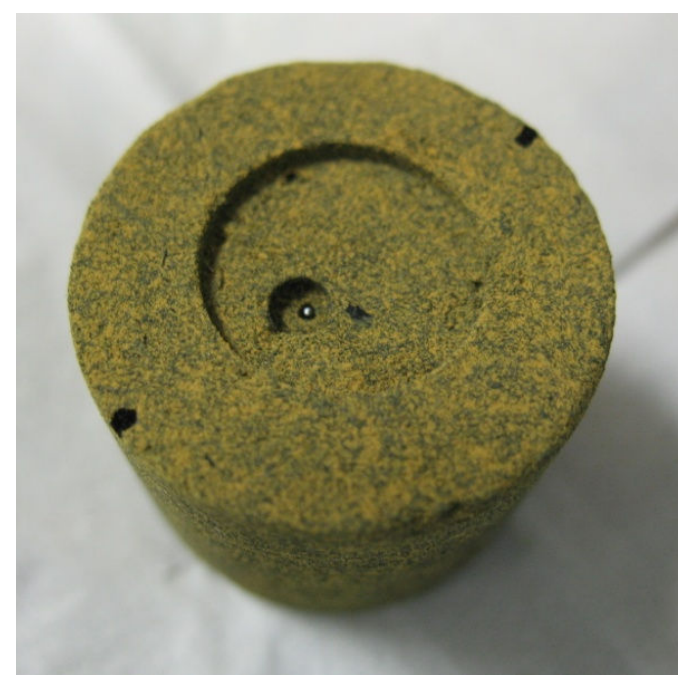

Figure 3a. Sapphire window plug.

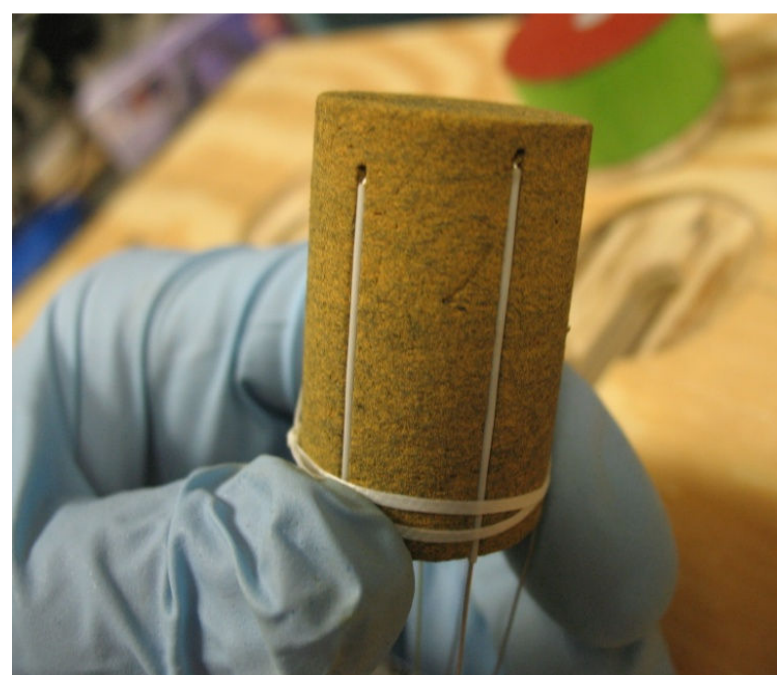

Figure 3b. Model thermocouple plug.

\section{Optical Instrumentation}

Because the primary objective was to demonstrate the feasibility of measuring off-body radiation with optical fibers embedded in ablative TPS materials, most of the fibers were coupled to silicon PIN photodetectors for timeresolved broadband measurements. We selected the Thorlabs PDA36A with a spectral response of 400-1100 nm for most of the models. The PDA36A has a built-in transimpedance amplifier and variable gain. The PDA25K GaP ultraviolet detectors with a spectral response of $150-550 \mathrm{~nm}$ were used for some model configurations. The detectors were mounted in the steel cable trays at the bottom of each sting arm, which provide access to any instrumentation leads that are run from the test models. Inserts were designed by ARC to rigidly hold the detectors in each tray but were abandoned because of difficulty in orienting the detectors without binding the optical fibers. The $600 \mu \mathrm{m}$ fibers required a large bend radius and were particularly difficult to position within the cable trays. The modular detectors had circular machined openings in front of the photodiode which accepted adapters for the fiber optic connectors. This resulted in the fibers oriented $90^{\circ}$ to the conduit leading to the sting arm. This added to the difficulty in fitting four detectors inside of the sting arm cable trays.

The spectrometer used was the commercial off the shelf (COTS) Czerny-Turner configured Ocean Optics HR4000CG high resolution unit. The grating has 300 lines $/ \mathrm{mm}$ and in combination with the $10 \mu \mathrm{m}$ slit provides a spectral resolution of $1.1 \mathrm{~nm}$ full-width-half-max (FWHM). The detector is a Toshiba TCD1304AP linear charged coupled device array with 3636 pixels. The instrument was connected to the models through a 25 -foot long, $400 \mu \mathrm{m}$ diameter fiber patchcord. The fiber was fed into the test chamber through an instrumentation plate with a Conax gland fitting for sealing. The fiber was routed to the sting arm traversing stage and fished into the cable tray through the conduit from the rear. Once the patchcord was in place, it was left throughout the testing and connected to the various model pigtails using a mating sleeve. The AHF sting arms are fitted with potentiometers that provide analog signals proportional to position. The spectrometer COTS software was set up for external triggering by the rising edge sting position signal and worked properly during pre-run testing. However, the first attempt to collect data for a run failed due to premature triggering on noise which left insufficient time to reset the software before the model was in position. The external trigger was abandoned and subsequent attempts were initiated manually by headset communication. Figure 4 a shows a simple schematic of the geometry of the observed line of sight. 


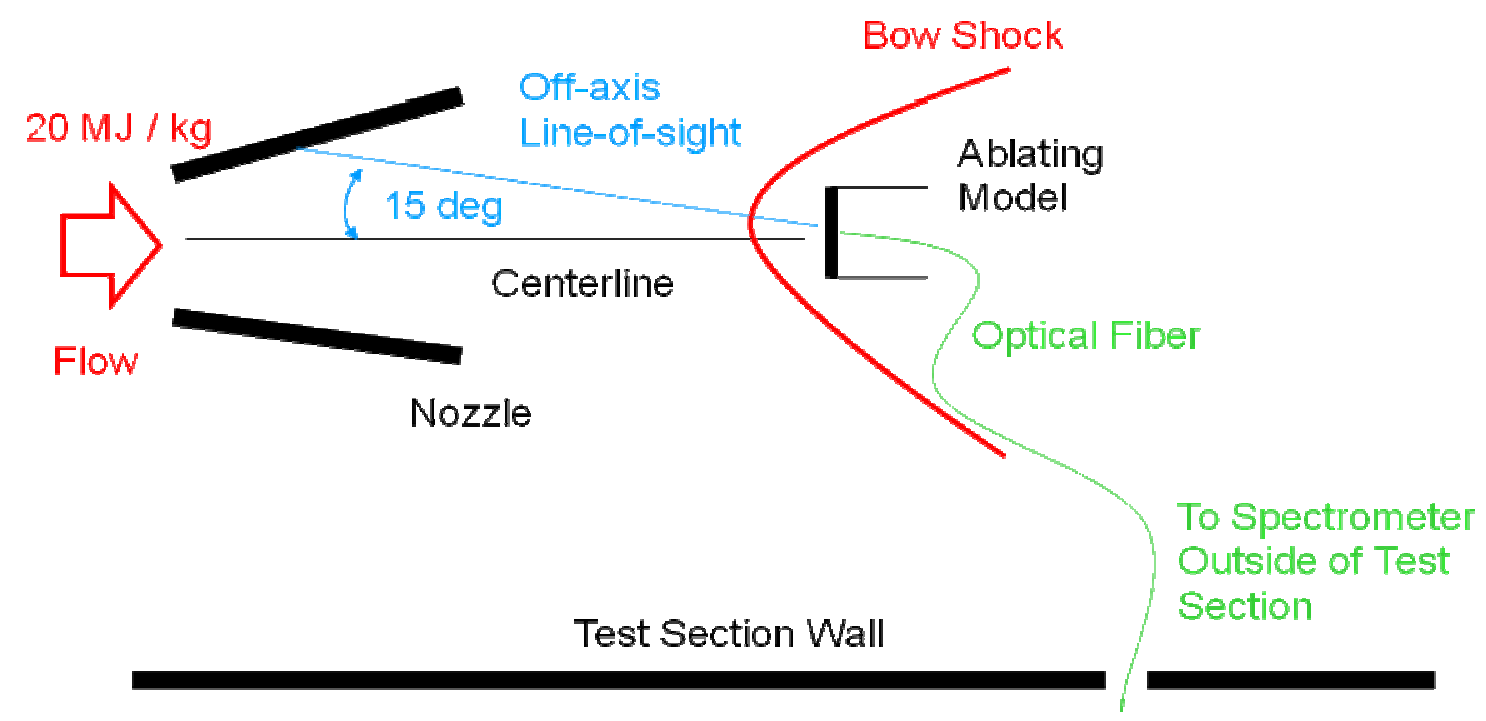

Figure 4a. AHF test schematic for optical measurement of gas temperature.

\section{Testing and Results}

Results of the initial demonstration tests show that the embedded fibers survive and operate during the first several seconds of model exposure to the flow. This approach of Figure 2 allowed data acquisition of optical spectra over the range $300-900 \mathrm{~nm}(0.04 \mathrm{sec}$ integration time at $8 \mathrm{~Hz}$ repetition rate). These results illustrate the potential of the approach for use in an instrumented aeroshell. When calibrated to absolute spectral radiance, such high resolution spectra will enable analysis of radiating species of both the shocked free stream gas and the blown gas of the ablator as a function of time during atmospheric entry. Additionally, an instrumented TPS model for arc jet testing can be used as a research tool on its own, providing critical data on the spectral radiance of free stream and blown gas species.

Each model was inserted into the flow for a maximum of $40 \mathrm{sec}$ unless the run was called by the Principal Investigator. All fourteen models were tested but not according to the original test matrix. While the first two runs were intended to allow tweaking of the detector gains to optimize signal to noise, a pre-test bond failure forced a rework of three of four models set to be tested in the calibration runs. A gain of $20 \mathrm{~dB}$ on the detector amplifiers was used for most of the models, while a gain of $50 \mathrm{~dB}$ was used on the UV detectors because of their lower responsivity.

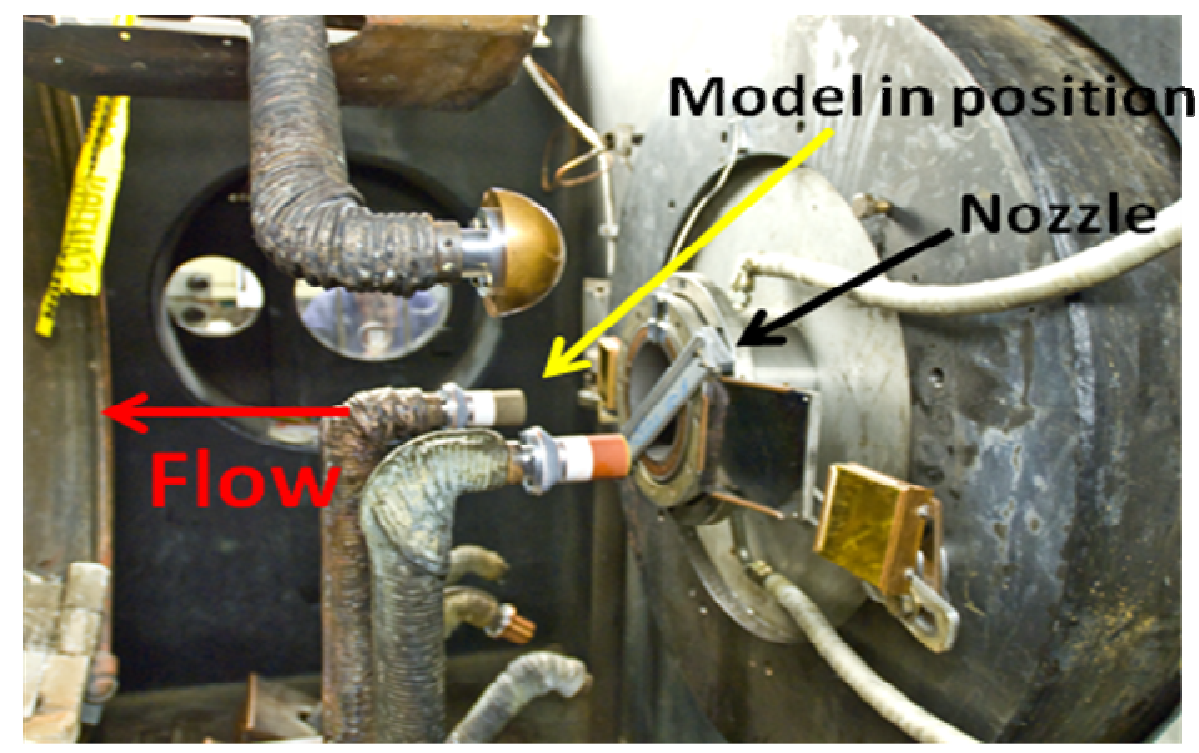

Figure 4b. Photograph showing arc jet nozzle and orientation of the test model.
The models were generally oriented on the stings with the shallowest counterbore at the twelve o'clock position.

Figure $4 \mathrm{~b}$ shows two of the test models installed on two of the five stings in the test chamber facing the arc jet nozzle. A hemispherical copper calorimeter (used for flow calibration before the models are inserted) is also shown on a sting arm above. 


\section{Special Model Configurations}

Model LX8 was fitted with a plug containing a $300 \mu \mathrm{m}$ fiber at a depth of $0.29 \mathrm{in}$. with a sapphire window above but not in contact with it. Although the model did not hold up well during the test, $22 \mathrm{sec}$ of radiometric data were recorded until the model was extracted. The access hole in the face above the window began to open up eventually exposing most of the plug before the model was removed. The noise in the data could be a result of the material passing in front of the fiber as it came apart. The amplitude of the data was on the order of six times that of an identical fiber/amplifier configuration on another model. The window was dislodged from the model upon extraction but there is no evidence in a facility video that the window cracked or left the model during the data acquisition period. However, the video was taken at a slow frame rate and some detail was lost. The window may have fallen from the model and shattered on the cold floor when the sting arm was lowered in a down and forward manner. Pieces of the window that were found had a milky appearance. Model LX6 was machined with blind holes behind (under) the fiber recesses with extremely thin walls of material left in front of the fibers. The walls were measured electromagnetically to be on the order of 0.014 and 0.029 in. thick respectively. The two fibers should have responded at different times as the material receded, but the experiment was a disappointment. One of the detectors saturated in $\sim 0.2 \mathrm{sec}$. It is possible that the detector gain switch could have been accidently moved to a higher setting but records do not indicate that. It is also possible that the switch could have opened in between two settings, resulting in infinite gain. The other channel behaved normally but also responded immediately resulting in a failed experiment. It should be noted that extreme care was taken not to puncture the walls during fiber installation leaving an uncertain distance between the fiber tip and the wall.

Figure 5 shows the data obtained from Model LX11, which was fitted with a plug instrumented with three TCs and a single fiber. The data from the three TCs show the time lag of the heating at different depths at the center of the model. The TCs were installed at depths of $0.1,0.2$, and 0.45 in. respectively. Figure 6 (Model LX10 fitted with four fibers and no TCs) shows repeatedly that the useful fiber observation time is longer for deeper recessing of the fibers. One of the fibers (turquoise trace) shows poor transmission and may have suffered damage during installation since the same amplifier unit appears to have worked properly on other runs. It is unlikely that debris in front of the fiber could have caused the attenuation; any debris would have burned away, and the fiber signal would have eventually saturated, as did the others. The black body emission from the molten fiber tips (see Figure 7a) that drives each detector into saturation, depending upon fiber depth, is apparent and repeatable.

LX11 THERMOCOUPLE/FBER RESULTS

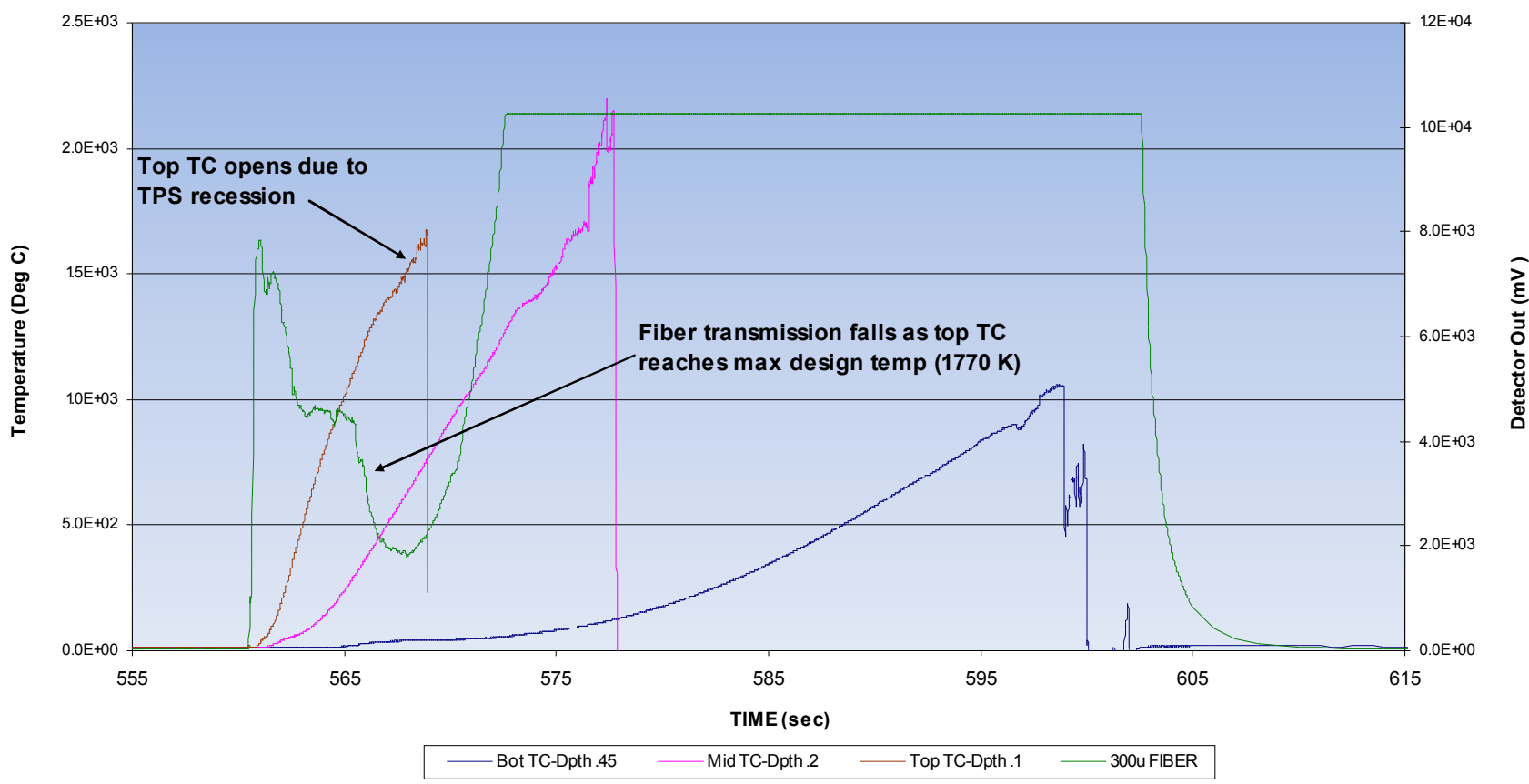

Figure 5. Temporal history of Model LX11 optical transmission through fiber (green) and three simultaneous TC outputs (brown $=$ shallowest, pink $=$ mid-depth, blue $=$ deepest TC). 


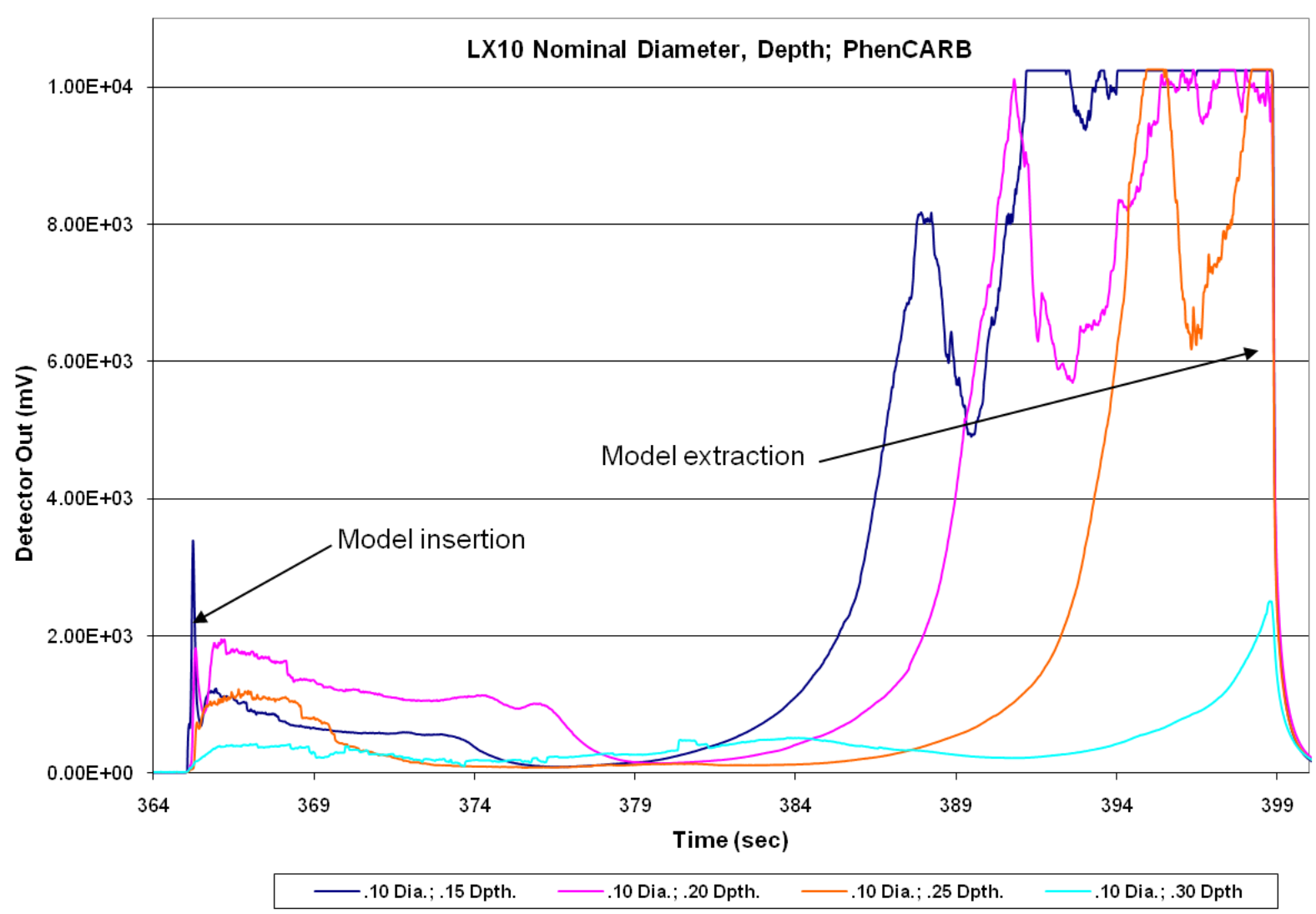

Figure 6. Model LX10 (PhenCarb 28) response lag of fibers recessed at different depths.

The radiometric (broadband) data collected during the tests are difficult to interpret. Most of the results are not repeatable in amplitude and are relatively noisy. There are many similarities in the data sets, however. Some of these are as follows:

a) The order of port insertion and extraction from the flow is apparent and indicates the physical orientation of the models. This is seen as time lag in the signal between different channels during insertion.

b) At the moment of insertion, the data was at its useful peak amplitude and then slowly tapered off to a minimum after approximately $10 \mathrm{sec}$. At that time, a slow $(4-5 \mathrm{sec})$ smooth transition occurred where the signal ramped up and saturated the detector until model extraction. This can be seen in Figure 6. The depth of the fiber dictates when this transition occurs. The deepest fiber transitions several seconds before the next deepest and so on with the entire time being on the order of $15 \mathrm{sec}$ in one data set. It is noteworthy that once the signal returns to the linear operating region, the fiber is still transmitting and indicates the order of removal from the flow. Speculation that the signal is due to incandescence of the fiber end is consistent with the observation that both spectral and radiometric signals (Model LX3) become very weak at the time of this transition.

c) Much of the data shows a significant transient peak at the point of model insertion that is too slow to be an electronic artifact, such as impedance mismatch. This usually occurs on the first port to enter the flow. Often there are weaker replicas from adjacent fibers.

A summary of results related to the different fiber optic configurations that were compared is as follows:

1. No advantage was gained by the smallest diameter $(0.05$ in.) counterbores.

2. Shallower fibers provided more signal than deeper fibers.

3. Deeper counterbores prolonged the life of the fiber.

4. Larger fibers withstood the environment better than smaller ones, possibly due to better heat conduction. For example, the $600 \mu \mathrm{m}$ fibers ended the runs protruding slightly above the charred model front surface.

5. A protective sapphire "storm window" appears to have given an advantage to fiber performance and life.

6. Multiple (3) small $(300 \mu \mathrm{m})$ fibers in a single recessed hole performed poorly compared to a single large fiber. 


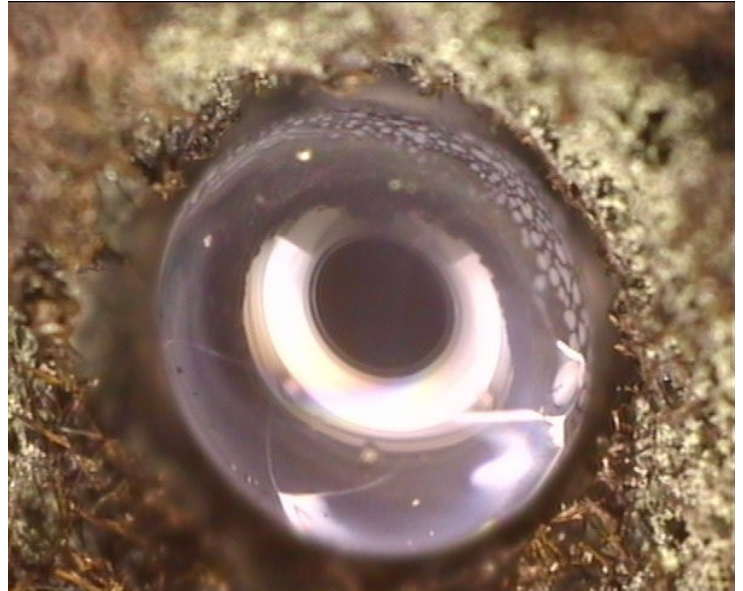

Figure 7a Post-test photograph of $600 \mu \mathrm{m}$ fiber.

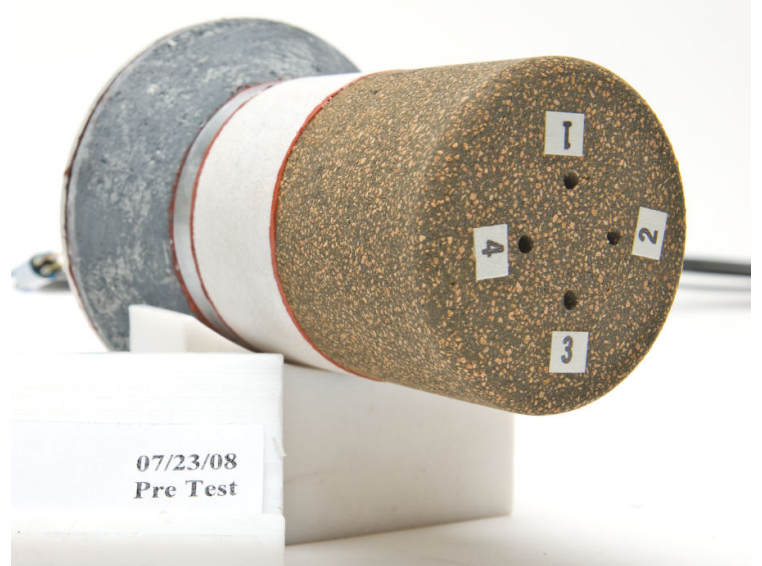

Figure 7b Pre-test photo of Model LX10 (PhenCarb 28).

\section{Measurement of Excitation Temperature}

The photograph of Figure 8 shows the charred condition of Model LX3 after a 35-sec exposure in AHF, which can be compared to the pretest photograph of Figure $2 \mathrm{~b}$. As shown in Figures 2 and 7, the fibers are located inside the model and observe upstream through the entire thickness of the gas cap into the freestream. The fibers are angled slightly off the flow centerline to avoid viewing the intense emission from the arc heater (see Fig. 2a). The acquired spectra are through the hot gas centerline. averaged over a line-of-sight cap adjacent to the flow

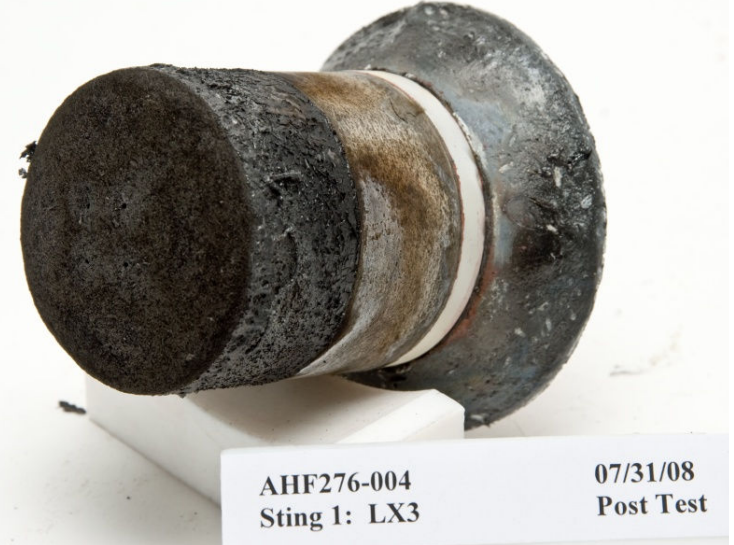

Figure 8. Post-test photograph of Model LX3.

The spectra of Figure 9 were acquired from the test with Model LX3 and show the evolution of the optical emission over a $1 / 4 \mathrm{sec}$ time period, just after insertion of the model into the flow. Each of the three spectra is separated in time by $1 / 8 \mathrm{sec}$. The spectra of Figure 9 are not corrected for spectrally dependent losses and responsivity of the detector. When considering these losses, the continuum portion of the run-072 spectrum (brown) approximates a $5000 \mathrm{~K}$ blackbody and may arise from the heated fiber tip and/or blown particulates in front of the model. 
One spectrum of Figure 9 (run $072=$ brown trace) is repeated in Figure 10, where several atomic emission lines are identified along with their wavelengths. We omit molecular band identifications, since some bands from different molecules overlap. The only ionized atomic species that we can identify is $\mathrm{Ca}^{+}$(pair of lines at about 395 $\mathrm{nm}$ ). Figure 10 also illustrates the weak $\mathrm{Cu}$ lines between 500 and $600 \mathrm{~nm}$. Trace amounts of copper in the free stream arise from vaporization of the arc heater electrodes. The relative intensities of the $\mathrm{Cu}$ lines were used to estimate a path-averaged electronic excitation temperature $\mathrm{T}_{\mathrm{e}}$ within the shock layer.

Temperature measurements using atomic $\mathrm{Cu}$ lines have been previously described ${ }^{19-21}$. Figure 11 shows a Boltzmann plot of the measured intensities of the line emission for four $\mathrm{Cu}$ lines from Figure 10 (versus the upper state energy level of the transition). This measurement has a $0.04 \mathrm{sec}$ integration time, acquired in the first few seconds of run 072. The vertical axis is the natural logarithm of the ratio of two products: the product of observed emission intensity I and the wavelength $\lambda$ of the line, divided by the product of the spontaneous emission coefficient $\mathrm{A}_{\mathrm{ij}}$ (i.e., the Einstein A coefficient) and the upper-state degeneracy $\mathrm{g}_{\mathrm{i}}$. The slope of the linear fit (solid line) gives a temperature of $4560 \pm 360 \mathrm{~K}(1 \sigma=68 \%$ confidence $)$.

LX3 INITIAL 3 SPECTRA

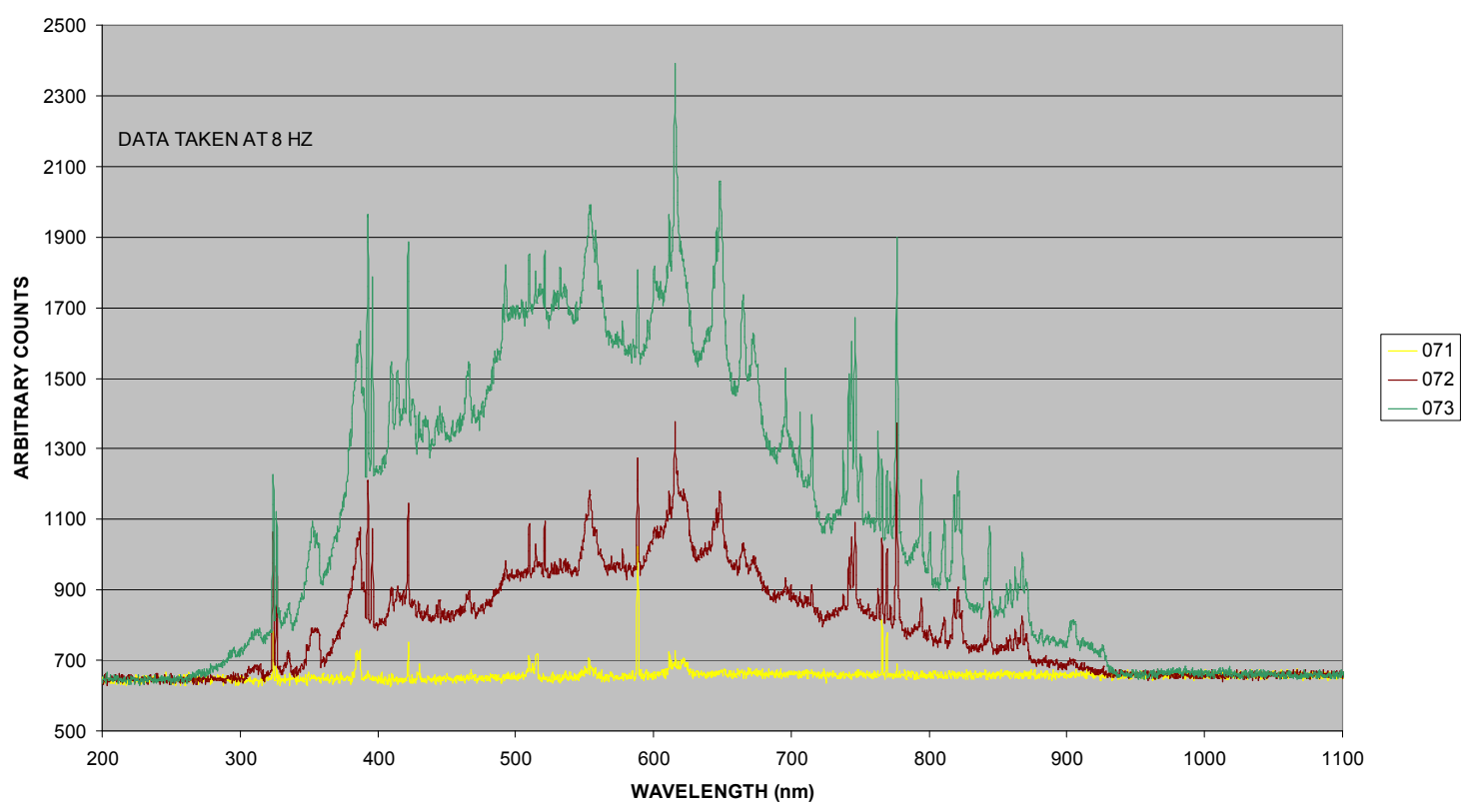

Figure 9 Three spectra at successively later times (acquired at $8 \mathrm{~Hz}$ ) during the first few seconds of a single run (yellow = earliest time $\&$ coldest gas, brown $=$ intermediate, and green $=$ latest time $\&$ hottest gas). 


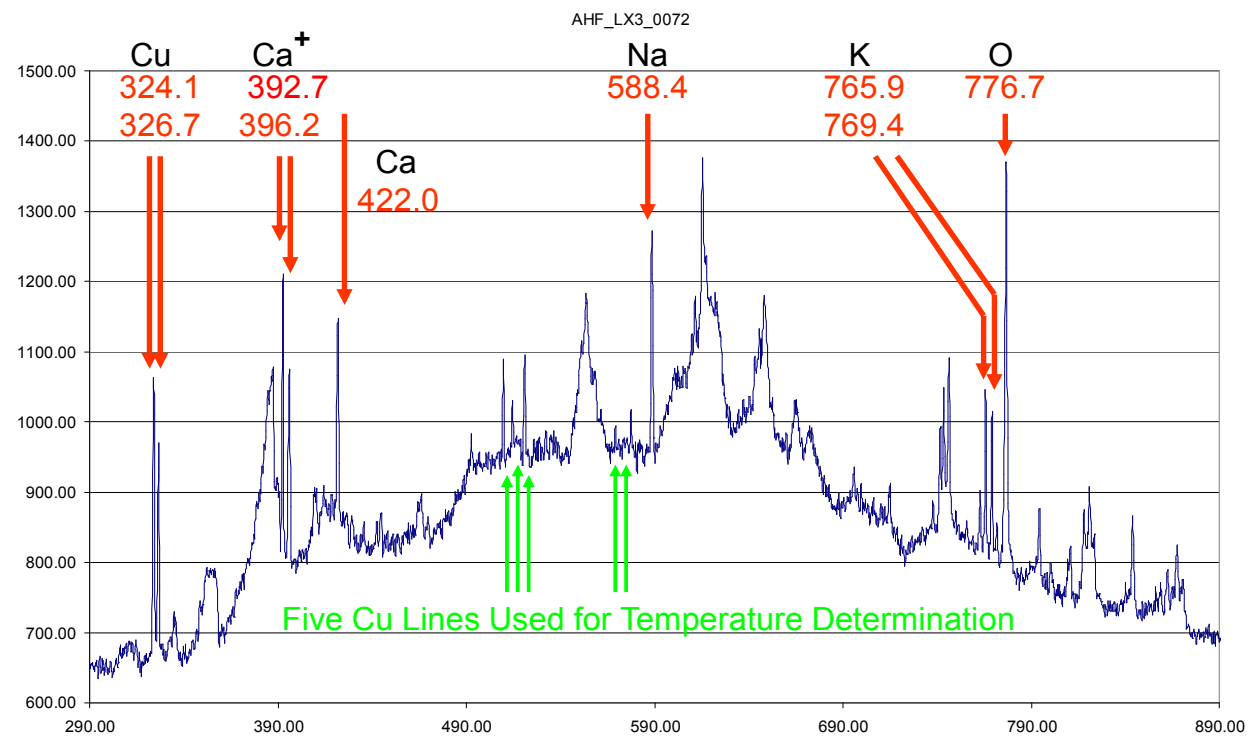

Figure 10 Visible-band spectra of hot gas cap identifying several atomic lines, including the $\mathrm{Cu}$ lines (green arrows) used for the path-averaged temperature measurement. Red numbers $=$ raw measured wavelengths $(\mathrm{nm})$ that give good agreement with known wavelengths for all 5 elements - if corrected upward by $\sim 0.6 \mathrm{~nm}$ (based on our laboratory calibration with $\mathrm{Hg}$ and $\mathrm{H}$ lamps).

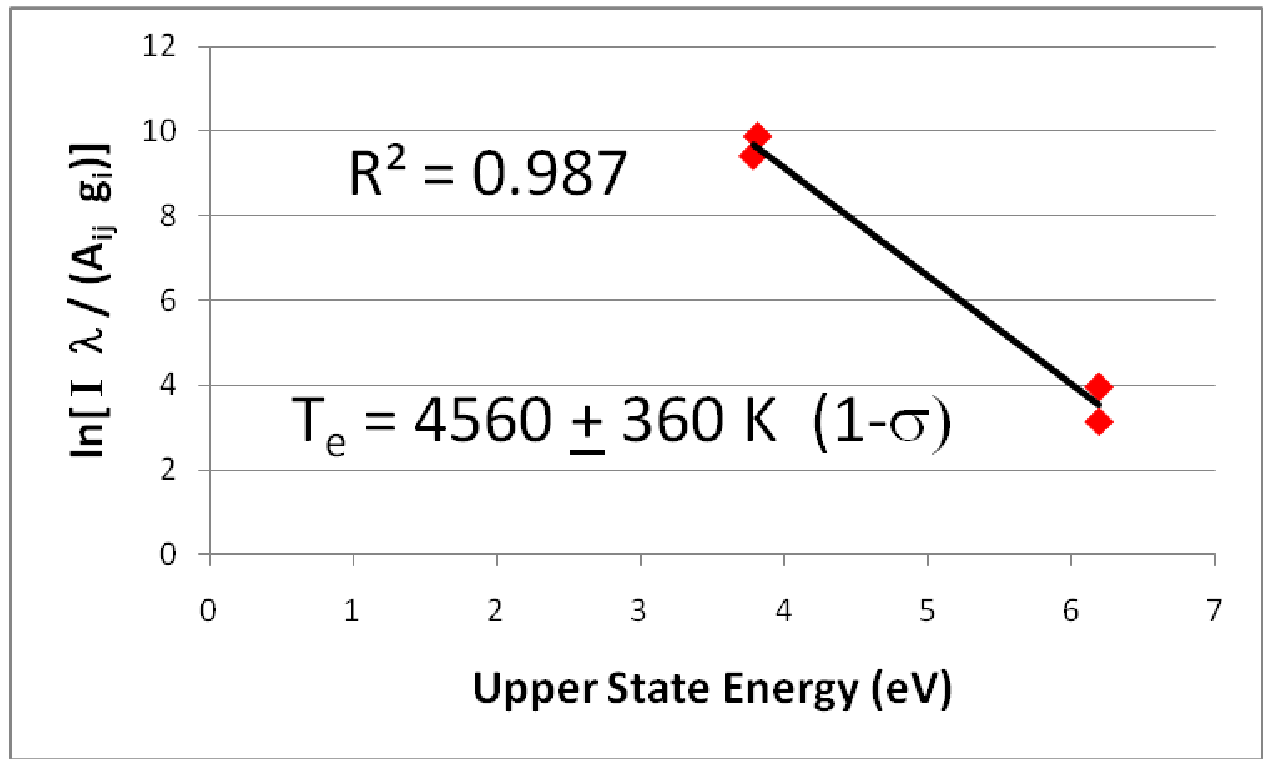

Figure 11 Boltzmann plot of measured atomic $\mathrm{Cu}$ line emission intensities as a function of upper state energy. 


\section{Summary}

We studied the feasibility of using optical fibers embedded in ablative TPS materials as a means to capture total and spectrally resolved shocked gas radiation ahead of an entry vehicle's aeroshell. The concept was demonstrated in a series of tests in the NASA Ames AHF arc jet. PICA and PhenCarb 28materials were used as the TPS materials for these tests. The experiments were the first, to our knowledge, use of optical fibers within ablative TPS materials. The bare ends of the embedded fibers were positioned at different depths from the surfaces of the arc jet models. The fibers were coupled either to photodiodes for total radiation measurements or to a miniature spectrograph for spectrally resolved measurements. The radiation signals were acquired as a function of time as the models were exposed to the $\sim 20 \mathrm{MJ} / \mathrm{kg}$ arc jet flow. The fibers retained their viability for the first several seconds after the models were injected into the flow. The spectra exhibited features characteristic of resonant radiation from atomic and molecular gas species and, possibly, thermal emission from ablation particulates and/or the end of the optical fiber itself. Emission from copper atoms present in the test gas was used to estimate a path-averaged temperature of the shock layer.

Although the extreme temperatures in the vicinity of the model surface damaged the ends of the optical fibers, further investigation is necessary to determine whether the fibers remain usable after prolonged exposure. We did not attempt to acquire spectroscopic data at times near the end of model exposure. Future efforts should include data acquisition throughout the model exposure period (and after) to determine if the fibers maintain viability.

The relatively low melting temperature of fused silica $(<\sim 2000 \mathrm{~K})$ limits the usefulness of this approach. In order to improve the practicability of the method, a means of protecting the fiber end would be required. We have shown that recessing the fiber end below the material surface prolongs its operational life. We also demonstrated that a protective sapphire window appears to improve performance, but this approach needs further experimentation and/or thermal modeling. Lessons learned from this first attempt will direct mechanical, thermal, and optical engineering efforts in the future.

The use of sapphire fibers is another interesting approach because of sapphire's higher melting point $\sim 2300 \mathrm{~K}$. One fundamental problem is that sapphire fibers typically have no cladding. Intimately embedding them in a TPS material will not work without an added cladding. This has been demonstrated experimentally in the literature but is not COTS. Because sapphire fibers are stiff and require a very large bend radius, it would be desirable to couple them to fused silica fibers a short distance away from the radiation source. This presents additional challenges that must be overcome to minimize attenuation. Since arc jet model (and reentry vehicle) bow shocks provide an extremely bright light source, however, exceptional transmission performance may not be necessary.

Future entry systems for human and robotic exploration missions will require forebody heat shields with ablative TPS materials. Flowfield-ablation coupling complicates development of computational tools used for analysis of candidate TPS configurations. Test data, whether from ground or flight testing, will be crucial for design tool development and validation. Emission spectra from the shock-heated and blown gases ahead of an ablative TPS material carry valuable information about the coupling of the flowfield aerothermodynamics with the ablator thermochemistry. The ability to capture these spectra in situ will provide a new, complementary source of test data. While significant technology development challenges remain, the results of this initial attempt at integration of fiber optics in ablative TPS warrant further investigation and investment.

Modern ground and flight test data for entry vehicles in relevant environments is the critical element validating our computational methods for application to the challenging space missions of the future. The present testing represents an attempt to modernize and resurrect flowfield measurement capabilities of the past. These successful measurements provide the foundation on which to build a low-cost, low-risk flight or flight test implementation.

\section{Acknowledgments}

We thank Johnny Fu, Imelda Terrazas-Salinas, Joe Mach, Walt Bruce, Hector Soto and Tom Hall for contributions to this work. 


\section{References}

${ }^{1}$ D. L. Cauchon, "Project Fire Flight 1 Radiative Heating Experiment,” NASA TM X-1222, 1966.

${ }^{2}$ D. L. Cauchon, "Radiative Heating Results from the FIRE II Flight Experiment at Reentry Velocity 11.4 Kilometers per Second," NASA TM X-1402, 1967.

${ }^{3}$ R. C. Ried, Jr., W.C. Rochelle, and J.D. Milhoan, "Radiative Heating to the Apollo Command Module: Engineering Prediction and Flight Measurement," NASA TM X-58091, 1972.

${ }^{4}$ D. B. Lee and W. D. Goodrich, "The Aerothermodynamic Environment of the Apollo Command Module During Superorbital Entry," NASA TN D-6792, 1972.

${ }^{5}$ P. A. Gnoffo, C.O. Johnston, and R.A. Thompson, "Implementation of Radiation, Ablation, and Free Energy Minimization Modules for Coupled Simulation of Hypersonic Flow," AIAA paper 2009-1399, 47th AIAA Aerospace Sciences Meeting, Orlando, FL, January 2009.

${ }^{6}$ J. H. Grinstead, D. M. Driver, and G. A. Raiche, “Optical Diagnostic Development for the Ames Arcjet Facilities," AIAA paper 2002-398, 40th Aerospace Sciences Meeting, Reno, NV, January 2002.

${ }^{7}$ A. Broc, V. Joly, J.-P. J. Lafon, and C. Marmignon, "Nonequilibrium Radiative Hypersonic Flow: Aerospace Applications," Astrophys. \& Space Sci., Vol. 260, 1998, pp. 29-43.

${ }^{8}$ V. Lago, A. Lebéhot, M. Dudeck, S. Pellerin, T. Renault, and P. Echegut, "Entry Conditions in Planetary Atmospheres: Emission Spectroscopy of Molecular Plasma Acrjets," J. Therm. \& Heat Trans., Vol. 15, 2001, pp.168-175.

${ }^{9}$ G. A. Raiche and D. M. Driver, "Shock Layer Optical Attenuation and Emission Spectroscopy Measurements During Arc Jet Testing with Ablating Models," AIAA paper 2004-825, 42nd AIAA Aerospace Sciences Meeting, Reno, NV, January 2004.

${ }^{10}$ J. Fujita, M. Mizuno, K. Ishida, and T. Ito "Spectroscopic Flow Evaluation in Inductively Coupled Plasma Wind Tunnel," J. Therm. \& Heat Trans., Vol. 22, 2008, pp. 685-694.

${ }^{11}$ K. Komurasaki, J. Kasahara, S. Yano, and T. Fujiwara, "Radiation in a Hypersonic Shock Layer Generated Around a Projectile," Shock Waves, Vol. 5, 1995, pp. 217-224.

${ }^{12}$ G. Palumbo, R. A. Craig, E. W. Whiting, and C. Park, "Measured Specific Intensity From 130 to $190 \mathrm{~nm}$ at the Stagnation Point of a Model in an Arcjet Flow of 7.8 km/sec," J. Quant. Spec. Radiat. Trans., Vol. 57, 1997, pp. 207-236.

${ }^{13}$ G. Walberg, W. L. Wells, W. M. Houghton, and R. E. Midden, "PERF: A New Approach to the Experimental Study of Radiative Aerodynamic Heating and Radiative Blockage by Ablation Products," AIAA paper 1974-1272, CASI/AIAA Joint Meeting, Toronto, Canada, October 1974.

${ }^{14}$ P. W. Erdman, E. C. Zipf, P. Epsy, C. Howlett, D. A. Levin, R. Loda, R. J. Collins, and G. V. Candler, "Flight Measurements Of Low-Velocity Bow Shock Ultraviolet Radiation,” J. Therm. \& Heat Trans., Vol. 7, 1993, pp. 37-41.

${ }^{15}$ P. W. Erdman, E. C. Zipf, P. Epsy, C. Howlett, C. Christou, D. A. Levin, R. J. Collins, and G. V. Candler "In Situ Plume Radiance From Bow-Shock Ultraviolet 2 Rocket Flight,” J. Therm. \& Heat Trans., Vol. 7, 1993, pp.704-708.

${ }^{16}$ J. Kurtz, R. Palmer, A. Thomas, T. McIntyre, and T. Steinberg, "Bow-Shock Radiation on Model Surfaces in Super-Orbital Flows," paper 7990, 21 st International Symposium on Shock Waves (ISSW 21), Great Keppel Island, Australia, July 1997.

${ }^{17}$ A. Balter-Peterson, F. Nichols, B Mifsud, and W. Love, "Arc Jet Testing in NASA Ames Research Center Thermophysics Facilities," AIAA paper 1992-5041, AIAA 4th International Aerospace Planes Conference, Orlando, FL, December 1992.

${ }^{18}$ D. G. Fletcher and D. J. Bamford, “Arcjet Flow Characterization Using Laser-Induced Fluorescence of Atomic Species," AIAA paper 1998-2458, 7th AIAA/ASME Joint Thermophysics and Heat Transfer Conference, Albuquerque, NM, June 1998.

${ }^{19}$ J. W. Schuttevaer, M. J. De Bont, and T. H. Van Den Broek, "Determination of the Relative Transition Probabilities of Some Triplet Lines in the Atomic Spectra of Calcium and Strontium," Physica, Vol. 10, 1943, pp. 544-552.

${ }^{20}$ D. H. Greenshields, "Spectroscopic Measurements of Gas Temperature in Arc-Heated Jets and Tunnels," NASA TN D$1960,1963$.

${ }^{21}$ N. Moriyama, J. Sekikawa, and T. Kubono, "Spectroscopic Temperature Measurement of Breaking Arcs Near Anode and Cathode Surfaces of Copper Contacts,” IEICE Trans. Electron., Vol. E89-C, 2006, pp. 1141-1146. 